\title{
Paradoxical mechanics for creation of adaptive gear mechanism - Devoted to International Day of Science
}

\author{
Konstantin Ivanov A. ${ }^{1,2, ~ *, ~ B u l a t ~ H i s a r o v ~}{ }^{1, ~ * ~}$ \\ ${ }^{1}$ Chair "Control Systems for Aerospace Engineering”, Almaty University of Power Engineering and Telecommunications, Almaty, \\ Kazakhstan \\ ${ }^{2}$ Laboratory of Adaptive Mechanisms, Institute of Mechanics and Machine Science MON of Republic Kazakhstan, Almaty, Kazakhstan
}

Email address:

ivanovgreek@mail.ru (Konstantin I. A.), hbd@mail.ru (Bulat H.)

\section{To cite this article:}

Konstantin Ivanov, Bulat Hisarov. Paradoxical Mechanics for Creation of Adaptive Gear Mechanism - Devoted to International Day of Science. American Journal of Mechanics and Applications. Special Issue: Adaptive Transmissions. Vol. 2, No. 6-1, 2014, pp. 1-4. doi: 10.11648/j.ajma.s.2014020601.11

\begin{abstract}
Paradox of the mechanism theory has been detected in connection with attempts of practical use mechanism with two degree of freedom and one entry for achievement of power adaptation. According to the mechanism theory and machines such mechanism is statically indefinable. However recently there were patents and publications with the description of efficient two mobile adaptive mechanisms with one entry. Paradox of the mechanism theory consists in the following: the kinematic chain with two degree of freedom and one entry containing the closed contour is statically and kinematically definable. In Ivanov's works the proof of definability of two mobile kinematic chain motion is resulted on the basis of additional analytical constraint by a virtual works principle. A certain geometrical image comparable to design there should match to analytical additional constraint. The present work is devoted to disclosing of a geometric constraint of parameters of kinematic chain with two degree of freedom and to statement of the mechanism theory paradox in geometrical and an analytic form. Work is executed on the basis of regularities of mechanics and mechanism theory.
\end{abstract}

Keywords: Mechanism Science, Paradox, Force Adaptation

\section{Introduction}

Paradox of the mechanism theory has been detected in connection with attempts of practical use mechanism with two degree of freedom and one entry for achievement of selfregulation or power adaptation. According to the mechanism theory [1] such mechanism is statically indefinable as the degree of freedom number should be equal to input links number. However recently there were patents and publications with the description of efficient two mobile selfcontrolled mechanisms with one entry. Paradox of the mechanism theory consists in the following: the kinematic chain with two degree of freedom and one entry containing the closed contour is statically and kinematic definable.

In patents of Croquet [2] and Volkov [3] the idea of creation stepless adjustable transfer with constant cogging of toothed wheels in the form of the hydrodynamic converter and gear differential with two degree of freedom is presented. The mechanism with two degree of freedom and one entry in the form of gear differential is presented in Harris's patent [4].
Ivanov's patents $[5,6]$ provides the use of inertia properties at start-up and contains regularities of differential mechanism synthesis on the set range of transfer ratios.

Existing practical workings out in the form of patents for inventions are intuitive as a rule. They do not contain the theoretical proof of definability of two mobile mechanisms containing only one entry.

Ivanov has resulted the proof of definability of two mobile mechanism motion on the basis of virtual works principle [7]. Definability of motion of two mobile mechanisms with one entry leads to occurrence of basic new phenomenon - effect of power adaptation. However use of virtual works principle should be proved - the mechanism should have a certain additional constraint between links.

In works [8,9] additional constraint between links of two mobile mechanism with one entry has been theoretically proved. Analytical additional constraint represents a certain abstraction. Basic new aspect of constraint should have the certain geometrical image comparable to a design. The present work is devoted to disclosing of a geometric constraint of parameters of the kinematic chain with two 
degree of freedom and to statement of essence of paradox of the mechanism theory in geometrical and an analytic form. Work is executed on the basis of regularities of mechanics and mechanism theory.

\section{Research of Motion of the Kinematic Chain with Two Degree of Freedom}

The kinematic chain with two degree of freedom is presented in the form of a differential wheelwork with two carriers (Fig. 1).

The mechanism contains rack 0 , input carrier $H_{1}$, input satellite 2 , block of the central wheels $1-4$, block ring wheels $3-6$, the output satellite 5 and output carrier $H_{2}$. Toothed wheels form four link closed contour 1-2-3-6-5-4. Sizes of toothed wheels $1,2,3,4,5,6$ are defined by matching radiuses $r_{i} i=1,2,3,4,5,6$. Radiuses of carriers $r_{H 1}=r_{1}+r_{2}, r_{H 2}=r_{4}+r_{5}$.

To the right of mechanism the picture of speeds $V_{i} \quad i=1,2,3,4,5,6$ of mechanism links is presented.

Linear speeds $v_{i}$ are expressed through angular speed $\omega_{i}$ by formula $V_{i}=\omega_{i} r_{i}$. Linear speeds of carriers $V_{H i}=\omega_{H i} r_{H i}, \quad i=1,2$.

The initial picture of speeds is shown by full lines. Intermediate picture of speeds matches to change of initial parameters of motion at invariable input speed and is shown by dashed lines.

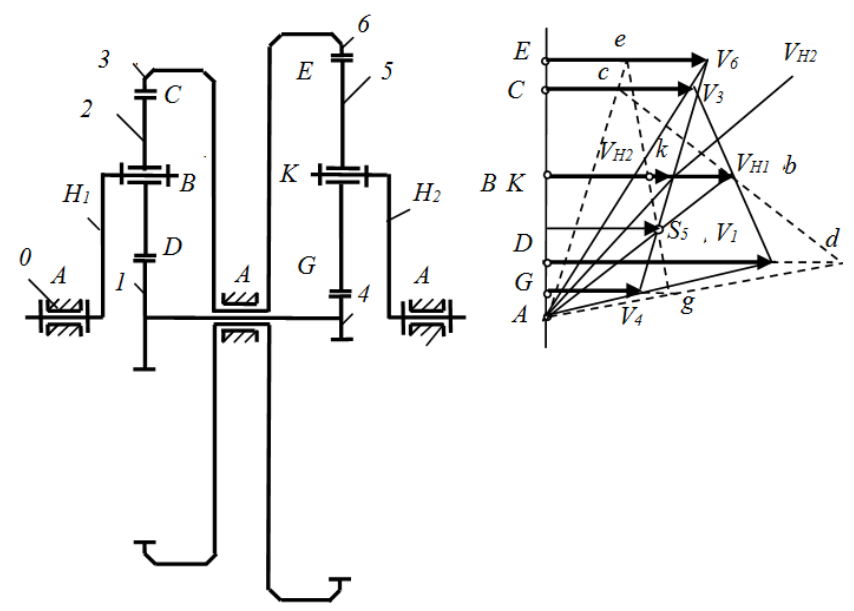

Fig 1. Gear differential mechanism and a picture of its speeds

On Fig. 1 it is visible that instantaneous center of turn $S_{5}$ of satellite 5 occupies invariable position on a line $A b$ of input carrier angular speed.

Let's prove that at constant angular speed of input carrier the $S_{5}$ of satellite 5 will occupy invariable position on a line $A b$.

Theorem: In the differential wheelwork containing two carriers, two satellites and two blocks of the central wheels the satellite has the constant instantaneous center of relative turn on the opposite carrier.
For the proof we will observe at first picture of speeds of the kinematic chain in inverse motion at motionless carrier $H_{1}$ (Fig. 2).

At motionless carrier $H_{1}$ linear speed $V_{H 1}=0$. The instantaneous center of turn $S_{2}$ of satellite 2 coincides with point $B$. We will run a line $c S_{2} d$ angular speed of satellite 2 . We will run a line $A c$ angular speed of a link 3-6 and it is found on it a point $e$ defining speed $V_{6}$ of mechanism point $E$. We will run a line $A d$ angular speed of link 1-4 and it is found on it a point $g$ defining speed $V_{4}$ of mechanism point $G$. We will run a line $e g$ angular speed of satellite 5 .
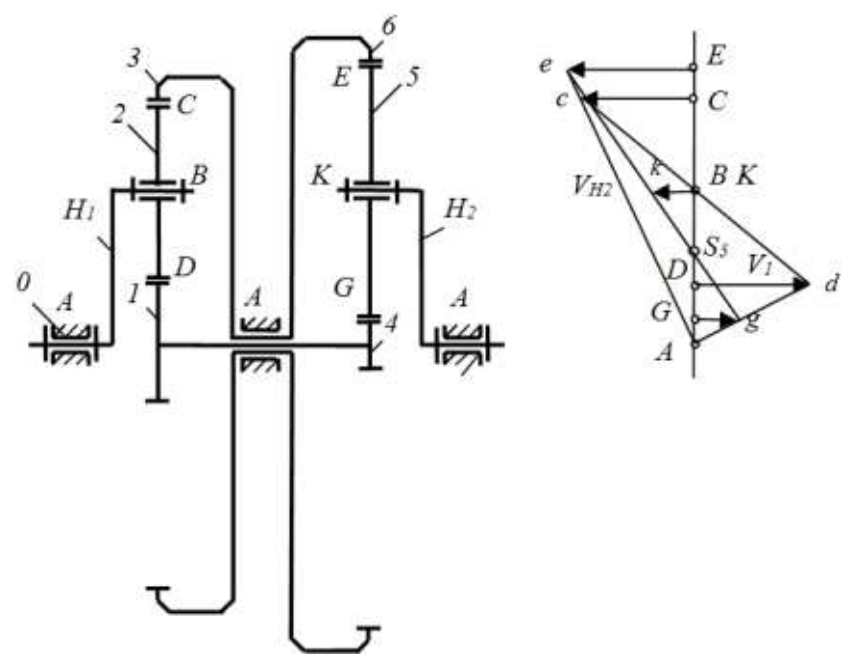

Fig 2. Gear differential mechanism and picture of it speeds in inverse motion

Intersection point $S_{5}$ of a line $e g$ with a vertical line of zero speeds is the instantaneous center of turn of satellite 5. Point $S_{5}$ is simultaneously instant centre of turn of satellite 5 concerning motionless carrier $H_{1}$. The position of point $S_{5}$ (size $y=B S_{5}$ ) can be defined through mechanism geometrics.

$$
y=r_{5} \frac{r_{1} r_{6}-r_{3} r_{4}}{r_{1} r_{6}+r_{3} r_{4}}
$$

Eq. (1) one-valued defines position of satellite 5 instantaneous center of turn $S_{5}$ in inverse motion concerning the motionless carrier $H_{1}$. In mechanism valid motion the point $S_{5}$ will move together with the carrier $H_{1}$ as it is shown in picture of speeds of moving mechanism (Fig. 1).

Hence the satellite 5 has the instantaneous center of relative turn on the opposite carrier $H_{1}$ as was to be shown.

The invariable position of the instantaneous center of relative turn $S_{5}$ on carrier $H_{1}$ defines an additional geometric constraint which occurs in the kinematic chain with two degree of freedom. However this additional constraint has no constructive performance. Mechanically the mechanism remains the kinematic chain with two degree of freedom and keeps the properties.

Eq. (1) expresses analytically this additional geometric constraint. As a result the kinematic chain becomes definable at presence only one input link. It means that at the set angular speed of input carrier it is possible to one-valued define speeds of all other links and mechanism points. 


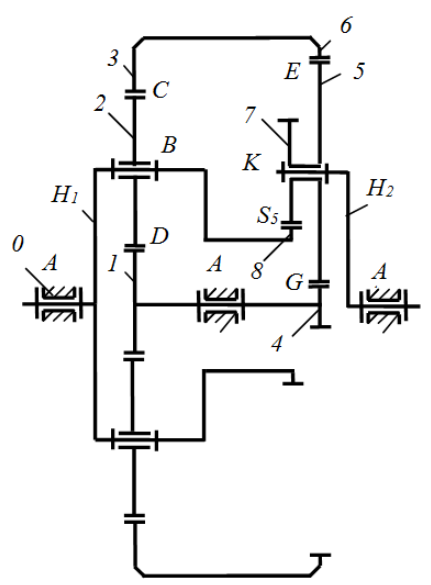

Fig 3. The conditional replacing mechanism with one degree of freedom

The additional geometric constraint leads to transformation of the initial kinematic chain to the conditional mechanism with one degree of freedom. The found regularity allows building replacing mechanism (Fig. 3) with the changed structure. However this constructive additional constraint is passive. Actually the degree of freedom number remains equal to two.

\section{Power Analysis of the Kinematic Chain with two Degree of Freedom}

In the presence of passive structural constraint and possibility of internal mobility of links of the closed contour according to picture of speeds (Fig. 2) it is necessary to recognize, first, possibility of definability of motion and, secondly, to install the reason of possible internal mobility of links of a contour.

The considered kinematic chain with two degree of freedom contains two links, having generalized co-ordinates (carriers $H_{1}$ and $H_{2}$ ) and the closed contour affiliated to them containing toothed wheels 1-2-3-6-5-4 and having zero mobility.

Statement of the power analysis problem.

Two generalized forces $M_{H 1}, M_{H_{2}}$ (moments of forces on carriers $H_{1}$ and $H_{2}$ ) are given.

It is necessary to define reactions in kinematic pairs.

The solution.

1) From carriers $H_{1}$ and $H_{2}$ on satellites 2 and 5 are transferred the reactions

$$
R_{H 1}=M_{H 1} / r_{H 1}, \quad R_{H 2}=M_{H 2} / r_{H 2}
$$

2) Reaction $R_{H 1}$ in point $B$ acts and reactions $R_{12}=R_{32}=R_{H 1} / 2=M_{H 1} / 2 r_{H 1}$ in points $D$ and $C$ acts on the satellite 2 .

We present condition of satellite 2 balance in the form of equality to null of the moments concerning the motionless instant centre of speeds $S_{20}$ (on Fig. 2 it is not shown).

$$
R_{12} \cdot D S_{20}+R_{32} \cdot C S_{20}=R_{H 1} \cdot B S_{20}
$$

3) It is analogous reaction $R_{H 2}$ in point $K$ acts and reactions
$R_{45}=R_{65}=R_{H 2} / 2=M_{H 2} / 2 r_{H 2}$ in points $G$ and $E$ act on the satellite 5 .

We present condition of balance of the satellite 5 in the form of equality to null of the moments concerning the motionless instant centre of speeds $S_{50}$ (on Fig. 2 it is not shown).

$$
R_{45} \cdot G S_{50}+R_{65} \cdot E S_{50}=R_{H 2} \cdot K S_{50}
$$

4) We will multiply the Eq. (3) on $\omega_{2}$. With the account $\omega_{2} \cdot D S_{20}=V_{1}, \omega_{2} \cdot C S_{20}=V_{3}, \omega_{2} \cdot B S_{20}=V_{H 1}$ we will gain

$$
R_{12} \cdot V_{1}+R_{32} \cdot V_{3}=R_{H 1} \cdot V_{H 1}
$$

Let's multiply the Eq. (4) on $\omega_{5}$. With the account $\omega_{5} \cdot G S_{50}=V_{4}, \omega_{5} \cdot E S_{50}=V_{6}, \omega_{5} \cdot K S_{50}=V_{H 2}$ we will gain

$$
R_{45} \cdot V_{4}+R_{65} \cdot V_{6}=R_{H 2} \cdot V_{H 2}
$$

Let's combine the Eq. (5) and (6)

$$
R_{12} \cdot V_{1}+R_{32} \cdot V_{3}+R_{45} \cdot V_{4}+R_{65} \cdot V_{6}=R_{H 1} \cdot V_{H 1}+R_{H 2} \cdot V_{H 2}
$$

The Eq. (7) contains the forces acting on the closed contour containing toothed wheels 1-2-3-6-5-4 and connected to two carriers (generalized co-ordinates). The left side of Eq. (7) represents the sum of powers (or works) internal forces of the closed contour. The right side of Eq. (7) represents the sum of powers (or works) external forces of the closed contour. We will consider kinematic pairs of the closed contour as ideal. Then the sum of works (or powers) internal forces of the closed contour is equal to null

$$
R_{12} \cdot V_{1}+R_{32} \cdot V_{3}+R_{45} \cdot V_{4}+R_{65} \cdot V_{6}=0
$$

From the Eq. (8) follows that the sum of works of external forces of the closed contour also is equal to null

$$
R_{H 1} \cdot V_{H 1}+R_{H 2} \cdot V_{H 2}=0
$$

Taking into account Eq. (2) we will gain

$$
M_{H 1} \cdot \omega_{H 1}+M_{H 2} \cdot \omega_{H 2}=0
$$

That is the sum of powers (or works) generalized forces is equal to null. It means that all considered kinematic chain is in equilibrium.

From Eq. (10) follows that one of the generalized forces should be negative. Than it is necessary to consider, for example, that $M_{H_{2}}$ is the moment of resistance on output carrier $H_{2}$ and $M_{H 1}$ is the driving moment on the input carrier $H_{1}$.

Thus it will appear that in the kinematic chain with two degree of freedom and with the closed contour only one link (input carrier $H_{1}$ ) can have generalized co-ordinate (independent angular speed $\omega_{H 1}$ ). Such kinematic chain will have the kinematic definability as Eq. (10) allows to define the output angular speed $\omega_{H 2}$ at the set moments $M_{H 2}$ and $M_{H 1}$.

Hence the kinematic chain with two degrees of freedom 
and with the closed contour at presence only one entry will have static and kinematic definability and will be the mechanism.

The mechanism with two degree of freedom possesses the surprising property outlawing from the Eq. (10)

$$
\omega_{H 2}=\frac{M_{H 1} \cdot \omega_{H 1}}{M_{H 2}}
$$

Eq. (11) expresses effect of power adaptation: at the set constant parameters of input power $M_{H 1}$ and input $\omega_{H 1}$ the output angular speed $\omega_{H_{2}}$ inversely proportional to the set variable output moment of resistance $M_{H 2}$. The mechanism creating effect of force adaptation was named as the adaptive mechanism.

Work of the adaptive mechanism begins with start at which the mechanism moves as a single whole with one degree of freedom (thanks to presence of a friction in the absence of an external resistance). After start the external moment of resistance translates the mechanism in a condition with two degree of freedom with internal relative motion of links in the presence of effect of force adaptation.

Presence of effect of force adaptation defines a discovery in the field of mechanics.

The discovery formula: Effect of force adaptation in the mechanic field consists that the kinematic chain with two degrees of freedom containing input link, output link and the closed mobile contour placed between them provides the motion of output link with a speed inversely proportional to loading on it at constant input power.

\section{Conclusion}

Paradox of the mechanism theory is solved. It is proved that the mobile closed mechanical contour of the kinematic chain with two degrees of freedom creates additional constraint and provides static and kinematic definability of chain at presence only one input. Such chain gets property of force adaptation.

The developed discovery allows providing the variable transfer ratio only at the expense of use of the closed contour and its properties without application of control means. Mechanical properties of the closed contour allow to provide the demanded transfer ratio independently, stepless and automatically.

\section{References}

[1] Levitsky N.I.: Theory of mechanisms and machines. M, the Science. Russia (1979).

[2] Samuel J. Crockett. Shiftless, continuously-aligning transmission. Patent of USA 4,932,928, Cl. F16H 47/08, U.S. Cl. 475/51; 475/47. USA (1990).

[3] Volkov I.V.: Way of automatic and continuous change of a twisting moment and speed of twirl of the output shaft depending on a tractive resistance and the device for its realization. The invention description to the patent of Russia RU 2234 626. Russia (2004).

[4] Harries John: Power transmission system comprising two sets of epicycle gears. Patent of Great Britain GB2238090 (A). UK (1991).

[5] Ivanov K.S., Yaroslavtseva E.K. : Way of automatic and continuous change of a twisting moment and speed of twirl of the output shaft depending on a tractive resistance and the device for its realization. The patent of Russia RU № 2398989. Russia (2010).

[6] Konstantin S. Ivanov: Almaty, KAZ - Owner of the registered sample. The name - Device of automatic and continuous change of a twisting moment - and changes of a corrected speed of output shaft depending on a tractive resistance. The deed on registration of the registered sample № 202012101 273.1. Day of Registration 02.05. (2012). German patent and firm establishment. Federal Republic Germany. (2012).

[7] Ivanov K.S.: The simplest automatic transfer box. WCE (2010). World Congress on Engineering 2010 (ICME). London, pp. 1179 - 1184. UK (2010).

[8] Ivanov K.S.theorem about balance of the closed contour.//the Mechanism theory and machines. The periodic scientificallymethodical log. №2 (16). 2010. A volume 8. The St.Petersburg state polytechnical university. With. $85-89$.

[9] Ivanov K.S. Paradox of mechanics - a basis of creation CVT. Transactions of 2-d IFToMM Asian Conference on Mechanisms and Machines Science. November 7-10, 2012, Tokyo, Japan. P. $245-264$. 